\section{Graphical User Interface}

O. Colhoun

Institut für Laboratoriumsmedizin, Klinikum Frankfurt

Höchst, Frankfurt am Main, Deutschland

Synonym(e) GUI; Graphische Benutzeroberfläche

Englischer Begriff Graphical User Interface

Definition Teil von Betriebssystem oder Anwendung, der dem Nutzer die Kommunikation mit dem Computer (z. B. der > Labor-EDV) ermöglicht.
Beschreibung Die Benutzeroberfläche vermittelt zwischen dem Anwender und den Programmen. Grafisch orientierte Benutzeroberflächen sind heute Standard nahezu aller Betriebssysteme und Anwendungsprogramme und werden deswegen manchmal als die eigentlichen Benutzeroberflächen angesehen. Der Bildschirm zeigt im Grafikmodus alle Aktionsmöglichkeiten mit Menüs und intuitiven grafischen Symbolen an, aus denen der Nutzer etwa durch Mausklick auswählen kann.

\section{Literatur}

Bibliographisches Institut \& F.A. Brockhaus (2003) Brockhaus Computer und Informationstechnologie. Bibliographisches Institut \& F.A. Brockhaus, Mannheim/Leipzig 